\title{
Cipango
}

Cahiers d'études japonaises

$24 \mid 2021$

Eugénisme dans le Japon moderne et contemporain

\section{Les mesures d'isolement des lépreux au Japon}

Leprosy isolation policies in Japan

Ishizaki Manabu

Traducteur : Traduit par Nathan Béridot

\section{OpenEdition}

Journals

Édition électronique

URL : https://journals.openedition.org/cipango/4223

DOI : 10.4000/cipango.4223

ISSN : 2260-7706

Éditeur

INALCO

Édition imprimée

Date de publication : 20 octobre 2021

Pagination : 17-32

ISBN : 9782858313969

ISSN : 1164-5857

\section{Référence électronique}

Ishizaki Manabu, «Les mesures d'isolement des lépreux au Japon», Cipango [En ligne], 24 | 2021, mis en ligne le 11 octobre 2021, consulté le 09 avril 2022. URL : http://journals.openedition.org/cipango/ 4223 ; DOI : https://doi.org/10.4000/cipango.4223

\section{(c) (7) (5)}

Cipango est mis à disposition selon les termes de la Licence Creative Commons Attribution - Pas d'Utilisation Commerciale 4.0 International. 


\title{
Les mesures d'isolement des lépreux au Japon
}

\author{
Leprosy isolation policies in Japan
}

\author{
IsHIZAKI Manabu \\ Professeur de droit constitutionnel à l'université Ryūkoku \\ Traduit par Nathan BÉRIDOT \\ Docteur de l'Inalco (IFRAE)
}

\section{Introduction}

Le 13 mars 2001, afin d'écouter les plaidoiries du procès des lépreux (procès de l'est du Japon), je me suis rendu à l'audience $\mathrm{n}^{\circ} 103$ du tribunal de district de Tōkyō.

Dès l'automne 1999, je m'étais engagé dans un travail de soutien des plaignants en tant que membre de l'Association de soutien des demandes indemnitaires des lépreux (Hansen byō - kokkabaishô seikyû soshô wo shien suru kai, ハン セン病・国家賠償請求訴訟を支援する会). Je m’y étais rendu avec des étudiants. Les témoignages et plaidoiries des plaignants, avocats et assistants que j'y ai entendus différaient considérablement de ce que j'avais appris dans divers documents. L'ampleur des dommages subis par les plaignants m’a décidé à leur cause aux côtés de l'association. Dans le même temps, je me suis efforcé de trouver dans la bibliothèque de l'université des travaux de droit constitutionnel portant sur la loi fondamentale intéressant les mesures d'isolement des lépreux 《 la loi de mesures de précaution contre la lèpre » (Rai yobō hō, らい予防法) 
18 Cahiers d'études japonaises n 24

(voir plus bas), mais rien n'existait sur la question. Je me suis questionné, en tant que chercheur, sur la raison de cette absence de sources universitaires en droit constitutionnel concernant la question des mesures d'isolement des lépreux. Dès lors, afin de mieux comprendre ce procès, je me suis rendu environ une fois par mois au sanatorium pour lépreux « Tama Zenshōen » (Kokuritsu ryōyōjo Tama Zenshōen国立療養所多磨全生園) de la ville de Higashimurayama東村山市 du département de Tōkyō東京都. En participant aux réunions de l'association de soutien qui se réunissait périodiquement dans la maison sociale de ce sanatorium, j'ai pu prendre part aux débats et notamment rédiger des bulletins. De plus, diverses conversations avec les résidents de ce sanatorium ont permis d'approfondir les échanges. Ce fut l'occasion de réfléchir en tant que citoyen à l'histoire des mesures d'isolement envisagées d'un point de vue de constitutionnaliste, d'approfondir la compréhension des dommages, mais surtout de se demander pourquoi les mesures d'isolement n'avaient pas (ne pouvaient pas) faire l'objet d'études constitutionnelles dans le Japon d'après-guerre. Ce fut également l'occasion de prendre conscience de la gravité des dommages subis par les plaignants. Par ailleurs plusieurs souvenirs amusants ont pu naître de cette période. En particulier, au printemps de chaque année, à la saison des cerisiers en fleurs, les hanami que j'ai partagés avec les plaignants resteront de bons souvenirs ${ }^{1}$.

Plusieurs fois, les jours de plaidoirie, j'ai fait le chemin entre le tribunal de Tōkyō et le sanatorium avec les plaignants et les assistants dans un bus aménagé par l'《Association de soutien ». Parmi les plaignants à ce moment déjà âgés, plusieurs personnes gardaient des séquelles de la lèpre et du travail dans le sanatorium (on faisait accomplir divers travaux aux pensionnaires !), et d'autres, longtemps isolées, n'avaient pas l'habitude des transports en commun. Pour ces gens qui avaient été coupés du reste de la société et avaient subi d'effroyables discriminations et préjugés, se déplacer jusqu'au tribunal n'était pas chose facile. C'est pourquoi aux procès de l'est du Japon, comme à ceux de l'ouest devant le tribunal de district de Kumamoto ou à ceux de la région de Setouchi devant le

1. TAKANAMI, 2003 : à la page 85 , je porte une veste blanche tout à droite de la photo qui contient des explications dans l'encart « Hanami avec les soutiens - le $1^{\text {er }}$ avril 2001, à Tama Zenshōen ». 
tribunal de district de Fukuyama, les plaignants avaient besoin du soutien des assistants pour se rendre au tribunal.

À travers ces activités de soutien, j'ai peu à peu fini par saisir l'étendue des dommages causés par les mesures d'isolement. Mais celle-ci m'apparaît insondable, et même quinze années après l'accueil des prétentions des plaignants par le tribunal de district de Kumamoto (11 mai 2001), il m'est toujours impossible d'affirmer avoir compris les souffrances endurées par les plaignants.

Mon incapacité à saisir l'étendue considérable de ces dommages s'est imposée comme une évidence grâce au témoignage d'An Sunin (安 逑壬), entendu dans une plaidoirie le 13 mars 2001.

\section{Mon bébé a été tué sous mes yeux}

Cette femme a été admise à l'âge de 16 ans dans un centre médical du département d'Okayama, sur une île perdue, en même temps que son père. Elle est tombée enceinte à l'âge de 17 ans, et à neuf mois de grossesse, a subi un avortement forcé. D'après son témoignage, cet avortement a été effectué par une infirmière et non un médecin. L'infirmière a saisi l'enfant et lui a dit que c'était un garçon. Elle a ensuite pris un bol en métal qui servait de réceptacle à des médicaments et des objets du dispensaire, puis a placé l'enfant à l'intérieur. Apparemment, elle lui a déclaré : «Regarde, c'est un garçon, regarde ! », en lui montrant l'enfant. Madame An m'a dit que l'enfant était bien vivant, qu'elle l'entendait pleurer et voyait bouger ses mains. L'infirmière l'aurait ensuite étouffé sous ses yeux avant de l'amener ailleurs. Madame An m'a dit qu'elle n'oublierait jamais les questions qu'elle s'était posées alors. Pourquoi devait-elle supporter une chose pareille ? Elle n'avait jamais tué personne ni jamais volé. On l'avait juste emmenée de force dans ce centre médical. En voyant le bébé qu'elle venait de mettre au monde se faire tuer, étouffé sous ses yeux, elle ressentait une profonde tristesse et un incroyable mépris à l'encontre des responsables².

Comment plaider à la place des victimes de telles expériences, comme madame An qui a subi un avortement forcé, ou plutôt a vécu le meurtre de son

2. HANSENBYŌ IKEN KOKUBAI SAIBAN ZENSHI HENSHŪ IINKAI, 2006, p. 378-379 ; HANSENBYŌ IKEN KOKUBAI SOSHŌ BENGODAN, 2003, p. 226-227. 
enfant ? Cet avortement forcé a été pratiqué sur elle en 1942-1943. C'était à l'époque de la loi sur les mesures d'isolement des lépreux (loi n ${ }^{\circ} 58,1931$ ), lors de la Seconde Guerre mondiale. Dans la loi eugénique nationale de 1940 (loi n 48, 1940), il n'existait aucune disposition légale sur la stérilisation ou l'avortement pour cause eugénique vis-à-vis des patients atteints de la lèpre. De ce fait, cet avortement a donc été pratiqué sur madame An en dehors de tout cadre légal.

En mars 2001, madame An résidait dans le sanatorium national pour lépreux « Tama Zenshōen ». À l'exclusion du moment qu'elle passa chez un proche après s'être évadée du sanatorium « Oku Kōmyōen », elle a été séquestrée pendant près d'un demi-siècle.

J'ai eu de la compassion quand je l'ai vue repartir dans le bus pour le Tama Zenshōen depuis le district de Tōkyō, après son témoignage au tribunal le 13 mars. J'ai entendu une phrase dans ce bus, qui m'a marquée plus encore que son témoignage. Elle m'a affirmé n'avoir dit qu'une infime partie des choses qu'elle souhaitait dire. Cette phrase, qui représente bien les dommages considérables engendrés par les mesures d'isolement, est restée gravée dans ma mémoire.

\section{Le procès de la lèpre}

L'agent responsable de la lèpre est le bacille de Hansen qui provoque une paralysie des nerfs périphériques, et cette maladie n'est que faiblement contagieuse. Ce nom vient du médecin norvégien Armauer Hansen qui l'a découvert ${ }^{3}$. Au Japon, puisqu'il fallait trouver un lieu pour prendre en charge les personnes atteintes de la lèpre, les mesures d'isolement se sont peu à peu renforcées à partir de la loi de prévention de la lèpre de 1907 (loi no 11, 1907). Le « mouvement antilèpre dans nos départements $\gg($ muraiken undō 無らい県運動), qui se donnait comme objectif de n'avoir aucun patient lépreux dans aucun département, a été lancé en 1929 dans le département d'Aichi, et s'est vite répandu dans tout le pays. Ce mouvement fut étendu par le gouvernement avec l'aide de la population, puis, accompagné du système d'internement obligatoire des patients lépreux, il

3. Auparavant au Japon on utilisait le terme rai $ら W$ pour désigner la lèpre mais les lépreux, faisant l'objet de discriminations et de préjugés, ne supportaient plus d'entendre ce terme. C'est pourquoi, nous utilisons aujourd'hui le terme de « maladie de Hansen ». 
fut renforcé par les régions. C'est ainsi qu'est né le préjugé selon lequel la lèpre était une maladie très contagieuse et facilement transmissible. En 1931, la loi de prévention contre la lèpre est entrée en vigueur (loi n 58,1931$)$ et treize centres médicaux nationaux des lépreux (nous parlerons de sanatorium) ont été créés sur des îles isolées de la mer intérieure ou sur des terres non habitées.

Après la rédaction de la Constitution du Japon de 1946, alors même qu' il y avait une avancée considérable dans la découverte de médicaments spécifiques pour le traitement de la lèpre, comme la Promine ou le DDS, les mesures d'isolement se sont poursuivies et une nouvelle loi de mesures de précaution contre la lèpre (loi $\mathrm{n}^{\circ} 241,1953$, qu'on appellera la nouvelle loi) a été rédigée.

La nouvelle loi avait pour but d'isoler uniquement les personnes susceptibles de transmettre la lèpre (article 6, alinéa 1) mais ce texte a été interprété différemment et presque tous les patients lépreux ont été visés par ces mesures d'isolement. De plus, cet isolement pouvait être imposé sur ordre de la région (article 6, alinéa 2). La nouvelle loi pouvait aussi limiter les sorties des patients du sanatorium (article 15), leur interdire d'être employés à durée déterminée (article 7), ou permettre la désinfection des endroits contaminés et de bâtiments également susceptibles d'être déplacés ou détruits (articles 8, 9, 18). Et de fait, les domiciles des patients ont été désinfectés. Ainsi, outre les patients, des familles entières devenaient objets de discriminations et de préjugés. D'autres lois ont été rédigées. En 1948, la loi de protection eugénique (la loi n 156, 1948) avait pour but de prévenir la naissance d'une mauvaise descendance (article 1), tandis que l'article 3 alinéa 1-3 de la même loi prenait également pour cible la descendance des personnes atteintes de la lèpre ou leurs conjoints, et autorisait la mise en place des avortements. De plus, l'article 14 alinéa 1-3 légalisait l'avortement des personnes atteintes de la lèpre ou de leurs conjoints ${ }^{4}$. Même avant que ces lois ne soient établies, sans se fonder sur aucune législation, des stérilisations ainsi que des avortements ont été pratiqués. Mais après l'établissement de ces lois, ces pratiques avaient une base légale. Ces lois indiquent que les stérilisations à but eugénique et les avortements sont mis en œuvre avec l'approbation de la personne ou de son

4. À propos de cette loi, Matsubara Yōko 松原洋子 écrit : « la loi de protection eugénique était une loi de discrimination des personnes handicapées ». MATSUbARA, 2002, p. 36. 
22 Cahiers d'études japonaises n 24

conjoint, mais en réalité les personnes se mariant et enfantant dans un sanatorium n'avaient pas le choix et étaient contraintes d'accepter l'avortement ${ }^{5}$.

La nouvelle loi ainsi que la loi de protection eugénique ont été abrogées en 1996. Mais aucune subvention n'a été accordée aux personnes qui étaient en isolement, et les responsabilités demeurent ambiguës.

De ce fait, le premier procès visant à une demande d'indemnisation auprès de l'État a été intenté au département de Kumamoto le 31 juillet 1998 par 13 personnes admises dans le sanatorium Hoshizuka Keiaien (Kokuritsu ryōyōjo Hoshizuka Keiaien国立療養所星塚敬愛園) du département de Kagoshima et le sanatorium Kikuchi Keifuen (Kokuritsu ryōyōjo Kikuchi Keifuen国立療養所 菊池恵楓園) du département de Kumamoto. Depuis, de nombreux plaignants qui ont été mis à l'écart de la société et ont vécu les mêmes dommages se sont ralliés aux autres plaignants, et le même procès a également été intenté dans les départements de Tōkyō et d'Okayama.

Le 11 mai 2001, le tribunal de district de Kumamoto a admis les prétentions de tous les demandeurs (l'État n'a pas interjeté l'appel) ${ }^{6}$. Cette décision fondée sur la récente loi « restriction des droits de l'Homme due aux mesures d'isolement » (kakuri kitei ni yotte motarasareru jinken no seigen, 隔離規定によってもたら される人権の制限) a été justifiée de la manière suivante :

Les restrictions aux droits de l'Homme contenues dans les dispositions de la nouvelle loi sur les mesures d'isolement ne constituent pas des interprétations raisonnables aux libertés de fixer sa résidence ou d'en changer. L'isolement des patients atteints de la lèpre, souvent pendant une très longue période, parfois pendant plusieurs années, est de nature à causer des dommages significatifs et décisifs sur la vie du patient. Certains malades ont été contraints d'interrompre leurs études, d'autres ont perdu leur emploi ou n'ont pas pu choisir leur profession, d'autres n'ont pu ni se marier, ni

5. On s'appuyait sur les mesures de « purification ethnique » et de « théorie de la honte nationale » avant et pendant la Seconde Guerre mondiale, puis après-guerre sur la théorie d'« État de culture ». Voir IshIZaKI, 2017.

6. Décision $\mathrm{n}^{\circ}$ 1748-30. 
construire un foyer, ont perdu une chance de mettre au monde et d'élever un enfant, ou encore ont été placés dans l'impossibilité de mener une vie familiale normale. Partant de ces effets, qui toutefois ont pu varier suivant les malades, il résulte qu'une atteinte significative a été portée aux possibilités de développement personnel auxquelles doit pouvoir prétendre une personne, et qu'une telle restriction des droits de l'Homme a pu être de nature à causer un préjudice à toute la vie sociale de ces personnes. Qu'en conséquence les restrictions aux droits de l'Homme ne se sont pas limitées aux seules libertés de fixer sa résidence et d'en changer, mais ont violé de manière plus large les droits fondamentaux protégés par l'article 13 de la Constitution.

En outre : « la gravité des conséquences des mesures d'isolement d'un patient requiert de faire montre de prudence dans leur mise en œuvre, c'est pourquoi elles ne sauraient être admises en présence d'autres méthodes plus appropriées pour le traitement des maladies infectieuses ni ne sauraient être autorisées que pour certaines maladies spéciales. »

Dans cette décision a été saisie l'importance de la restriction apportée aux droits de l'Homme par la nouvelle loi et par les mesures d'isolement. On y affirme que la nouvelle loi était anticonstitutionnelle dès sa promulgation car elle menait à une restriction démesurée des droits de l'Homme. « La promulgation de la nouvelle loi sur les mesures d'isolement vers 1960 a conduit à une situation aux conséquences disproportionnées, si bien que son caractère anticonstitutionnel ne faisait aucun doute. » De plus, « l'inaction du pouvoir législatif qui a conduit à ne réformer cette loi que tardivement en 1965 est de nature à engager la responsabilité de l'État pour illégalité sur le fondement de la loi sur la responsabilité administrative. » Ainsi, l'inaction du pouvoir législatif est anticonstitutionnelle et a conduit à une illégalité au sens de la loi sur la responsabilité de l'État (alors qu'était imposé par la Constitution le devoir de légiférer, le parlement ne l'a pas fait ; cette affaire met en lumière l'absence de loi antérieure à 1996 qui aurait permis d'abroger la loi sur les « mesures d'isolement contre les lépreux »). 
CIPANGO

24 Cahiers d'études japonaises nº 24

\section{Dommage collectif}

Comme l'indique la décision du tribunal de district de Kumamoto, « les effets de l'isolement ont pu varier suivant les malades ». Il est vrai qu'ils ont tous été victimes d'un dommage considérable, mais selon l'âge auquel ils ont été admis, la période, le sanatorium, leur sexe, l'ampleur des dommages diffère pour chaque patient. Dans ce procès contre les mesures d'isolement, il n'a pas été demandé une indemnisation au cas par cas pour chaque plaignant, mais une indemnisation collective du préjudice partagé par tous les plaignants, c'est-à-dire les dommages causés par l'isolement. La décision a fait droit à cette demande. Il s'agissait d'une stratégie des plaignants et des avocats qui souhaitaient, avant tout, que le cas soit rapidement jugé.

Certains plaignants n'ont pas donné leur vrai nom, mais le nom de leur sanatorium (afin d'éviter que leurs proches soient discriminés). Certains ont eu à payer des « coupons sanatoriaux » utilisables uniquement dans le sanatorium (afin d'éviter toute évasion du sanatorium) quand d'autres, en échange du droit de se marier au sein du sanatorium, ont eu à subir une stérilisation ou un avortement forcé. Il y a également des personnes qui ont reçu des traitements médicaux frauduleux, ou qui ont été forcées de travailler au sanatorium. D'autres ayant enfreint le règlement au sein du sanatorium ont été mises dans des cellules, dans des conditions inhumaines. Tous ces dommages engendrés par l'État n'ont pas été indemnisés par le procès : les énumérer tous un par un aurait pris des années.

Pour mieux donner une idée du dommage collectif, je vais vous présenter un autre plaignant. C'est une citation de la décision du tribunal de district de Kumamoto en mai 2001. Ci-dessous est reproduit l'avis du tribunal concernant les dommages causés à une plaignante (plaignante numéro 87) qui était en isolement sur une île perdue dans la mer intérieure, au sanatorium Ōshima Seishōen (Kokuritsu ryōyōjo Oshima Seishōen 国立療養所大島青松園)：

La plaignante numéro 87 est née dans le département d'Ehime en octobre 1948, mais a été élevée par sa grand-mère après la mort de sa mère qui était atteinte de la lèpre. Vers 1956, cette plaignante a contracté la lèpre et a reçu de nombreuses demandes du personnel départemental pour la convaincre de se rendre au sanatorium, 
mais la grand-mère refusait chaque demande au prétexte qu'elle ne souhaitait pas l'abandonner sur une île perdue. Toutefois le 16 avril 1957 (à l'âge de 8 ans), la plaignante a été admise au sanatorium Ōshima Seishōen. Dans le train qui l'a amenée à ce sanatorium, la plaignante a été exposée aux regards des autres. Puis, au moment de monter la colline pour atteindre le quartier des jeunes filles, lors de l'admission, elle avait l'impression d'entrer dans un tunnel obscur.

La plaignante, après avoir fini l'enseignement obligatoire du sanatorium, est allée au sanatorium de Nagashima Aiseien (Kokuritsu ryōyojo Nagashima Aiseien国立療養所長島愛生園) et est entrée au lycée Oku du sanatorium, à la classe Nīrada. Pendant ses études, sa grand-mère, la seule de sa famille qui lui apportait de l'aide, est décédée. Mais la plaignante n'a pas été conviée à ses obsèques du fait de ses proches qui s'opposaient à sa présence.

La plaignante, après avoir fini ses études sur place, est retournée à Ōshima Seishōen et s'est mariée avec un camarade du sanatorium en septembre 1970. Mais leur logement était séparé par une fine porte coulissante d'un couloir qui donnait sur deux autres logements occupés par des couples. L'endroit n'était pas du tout reposant. La plaignante est tombée enceinte deux fois, en 1970 et en 1990, mais le sanatorium ne lui laissant pas le choix, elle a été contrainte de subir un avortement.

La plaignante n'a jamais pensé quitter les lieux. Y étant depuis l'âge de 8 ans, un âge où l'on n'a pas encore développé de sociabilité, séparée de sa famille et vivant pratiquement jusqu'à son âge adulte sur une île perdue, nous pouvons dire que l'isolement est certainement la cause de son état mental actuel ${ }^{7}$.

En 2000, le 15 juin, la plaignante numéro 87 qui était en isolement à Ōshima Seishōen a été questionnée par son avocat. Il lui demandait « quelle aurait été votre vie si, en deuxième année d'école primaire, vous aviez bénéficié d'un

7. Décision nº 1748-104. J'ai moi-même mis le dernier paragraphe en italique. 
traitement médical chez vous à base de comprimés ? Cela veut dire que vous ne seriez jamais allée à Ōshima Seishōen mais que vous auriez été scolarisée dans une école primaire normale. » Elle avait alors répondu qu'elle aurait grandi et vécu normalement, comme un être humain normal ${ }^{8}$.

La décision de justice citée plus haut rend compte avec précision des dommages subis par la plaignante. C'est cela le dommage collectif, partagé et vécu par tous les plaignants.

\section{Avortements forcés et stérilisations sur les lépreux}

Comme indiqué plus tôt, la décision du tribunal de district de Kumamoto prononçant l'indemnisation ne couvre que le dommage collectif partagé par tous les plaignants, c'est-à-dire l'isolement ${ }^{9}$. Les dommages réellement subis sont incalculables. Nous savons également, grâce aux témoignages des plaignants, qu'il $\mathrm{y}$ a eu un nombre considérable de suicides au sein des sanatoriums. Il est tout à fait impossible d'énumérer les dommages causés aux plaignants de manière exhaustive. Je vous invite donc à consulter la décision du tribunal de district de Kumamoto ${ }^{10}$.

Je vais revenir sur certains dommages tels que les avortements forcés ou les stérilisations, car ils mettent en évidence la particularité du système d'isolement des lépreux au Japon.

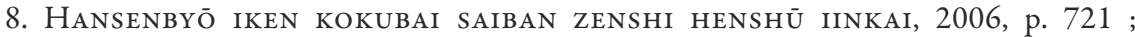
HANSENBYō IKEN KOKUBAI SOSHŌ BENGODAN, 2003, p. 144-149; TAKANAMI, 2003, p. $182-183$.

La plaignante $n^{\circ} 87$ s'appelle Shinohara Sumie (篠原 澄江). Son nom est cité dans les ouvrages ci-dessus. Durant les procès des lépreux, les noms des demandeurs ont été révélés à la société, si bien qu'ils craignaient qu'eux et leur famille fassent à nouveau l'objet de discriminations. Pour cette raison, plusieurs demandeurs ont agi anonymement. De ce fait, dans la décision est inscrit un numéro à la place de leurs noms.

9. Par exemple, voir la déclaration $n^{\circ} 2$ de Suzuki Tokiji (鈴木 時治) qui avait lui-même envisagé le suicide : HANSENBYō IKEN KOKUBAI SAIBAN ZENSHI HENSHŪ IINKAI, 2006, p. 232-233.

10. Sur la situation réelle des dommages, voir les écrits établis sur le fondement de la négociation dans les procès du tribunal de district de Kumamoto : NichibENREN HŌMU KENKYŨ ZAIDAN, 2007. 
Tout d'abord, quelques chiffres concernant les avortements forcés et les stérilisations qui ont été pratiqués sur les lépreux sous l'empire de la loi de protection eugénique entre 1949 et 1996 : 1551 cas d'avortements et 7699 cas de stérilisations. En 1996, l'année où cette loi a été abrogée, on recense 5 cas d'avortements forcés ${ }^{11}$.

Au sein des sanatoriums, les mariages entre lépreux étaient acceptés. Seulement, les hommes étaient souvent stérilisés et, si les femmes tombaient enceintes, elles étaient tenues de subir un avortement.

L'ancêtre du sanatorium Tama Zenshōen de Tōkyō qu'est l'hôpital Zensei (ouvert en 1909, son médecin-chef Mitsuda Kensuke 光田健輔était un personnage central du mouvement d'isolement des lépreux) pratiquait, au début de manière générale, l'isolement des hommes et des femmes. Mais à l'époque, les planches de bois ou les murets qui seuls permettaient d'enclore le compartiment des femmes ne suffisaient pas à les isoler. De ce fait, les naissances d'enfants au sein de l'hôpital étaient peu encadrées. Le médecin-chef Mitsuda, tout en acceptant qu'hommes et femmes aient des relations, a voulu stériliser les hommes pour empêcher la naissance des enfants. Cependant, cette stérilisation qui était une contrepartie du mariage et avait pour but d'empêcher les naissances et de mieux gérer l'établissement, avait également un autre but : «installer un mécanisme permettant de donner du sens à cette "autre vie" imposée aux patients en isolement au sanatorium ». Ainsi le mariage au sein du sanatorium a été redéfini et instrumentalisé ${ }^{12}$. L'on espérait également qu'accepter le mariage au sein du sanatorium empêcherait les évasions des patients. La pensée eugénique visant à l'anéantissement des lépreux, ainsi que l'idée illusoire que les patients, grâce aux règles établies et sous l'autorité du sanatorium, pourraient vivre une vie « heureuse », ont mené à cette forme de mariage avec condition de stérilisation.

La loi de protection eugénique soumettait à l'approbation de la personne concernée et du conjoint la stérilisation ou les avortements, mais en réalité, comme je l'ai écrit, ils y étaient forcés. Ainsi, la plaignante numéro 87 affirmait que « puisqu'il n'était pas question d'élever son enfant au sein du sanatorium,

11. Ibid., tome 1, p. 283-284.

12. Ibid., tome 1, p. 262-264. 


\section{CIPANGO}

28 Cahiers d'études japonaises nº 24

l'avortement était le seul choix qui me restait ». La plupart de ces personnes étaient dans le même cas.

Ce système qui acceptait que les patients en isolement forcé se marient tout en leur refusant le droit d'élever leurs enfants n'a pas d'équivalent. On ne saurait le comprendre sans connaître en amont la politique d'isolement à vie dans un sanatorium des lépreux ${ }^{13}$.

À ce propos, les embryons et les nouveaux-nés qui ont été avortés ont été conservés en tant qu'échantillons dans du formol. Ces échantillons ont apparemment été recueillis de 1924 à 1956 et d'après les rapports d'enquête sur les échantillons embryonnaires de 2005, 114 auraient été trouvés à la fin des recherches ${ }^{14}$. De plus, lors de la mise sous scellés des preuves en vue du procès du 30 octobre 2000, dans le bâtiment des dissections du sanatorium d'Oku Kōmyōen (Kokuritsu ryōyōjo Oku Kōmyōen 国立療養所邑久光明園) du département d'Okayama, plusieurs corps inanimés de nourrissons ont été retrouvés. De ce fait, l'avocat a prononcé ces mots devant le juge :

Ces spécimens sont des corps inanimés de nourrissons qui ont déjà la forme d'un être humain. On ne sait pas s'ils ont été avortés ou s'ils sont nés naturellement, mais ces spécimens ont été trouvés dans ces conditions. Sur cette étiquette est écrit naissance prématurée artificielle. C'est-à-dire qu'ils ont été mis au monde prématurément par quelqu'un. Au fond, il y a aussi deux autres corps de nourrissons. Trois, quatre et cinq corps, vous voyez la tête d'un nourrisson là-bas. Six, ah et ici aussi un autre, sept. Ces nourrissons ont été mis au monde de manière prématurée et artificielle ${ }^{15}$.

On voit qu'outre les avortements forcés, certains enfants ont été tués du fait de leur mise au monde prématurée et artificielle. Il s'agit donc d'une infraction. Cela coïncide également avec le témoignage relaté plus haut de madame An. De plus,

13. Fujino, 2001, p. 80-83.

14. Concernant les échantillons, voir le rapport détaillé dans Nichibenren hōMU KENKYŨ ZAIDAN, 2007, tome 2, p. 633.

15. HANSENByō iKen KoKUbai Soshō Bengodan, 2003, p. 240. 
si la défense a montré les pratiques de dissections lors de la période de mise sous scellés des preuves, c'est parce que ces preuves devaient être détruites sous peu. Mais nous ne pouvons affirmer qu'il n'y a pas eu d'échantillons embryonnaires même après 1956.

\section{Conclusion}

Comme on peut le constater, les dommages cachés subis par les patients sous ce régime d'isolement des lépreux sont considérables. Je n'ai ici relevé que quelques fragments qui représentent une infime partie des dommages. Du fait de leur immensité, ils paraissent impossibles à décrire dans leur totalité et nous n'avons pu en présenter qu'une fraction.

En conclusion, en tant que soutien ayant assisté aux procès des lépreux, je souhaiterais ajouter un mot. J'ai rencontré de nombreux plaignants à travers mes activités d'aide. Je me suis donné corps et âme dans cette association et il était très difficile, pour moi, d'entendre les récits de toutes ces expériences. Mais en réalité, j'ai aussi passé d'agréables moments lors de ces échanges avec les plaignants. La fête des cerisiers (hanami) que j'ai décrite en introduction en fait partie. Je finirai ce texte en vous décrivant l'un de mes souvenirs.

Je ne sais pas si c'était le jour où les plaignants, la défense et les soutiens s'étaient rassemblés devant la résidence principale du Premier ministre pour que l'État ne fasse pas appel de la décision du tribunal de district de Kumamoto, ou les jours qui suivaient, mais dans les environs du quartier Nagata, près de la résidence principale du Premier ministre, il m'était arrivé de prendre le train de retour avec l'un des plaignants, décédé aujourd'hui, monsieur Shibata Ryōhei ${ }^{16}$. L'un des soutiens, voyant que monsieur Shibata essayait de cacher l'écharpe sur laquelle était inscrit « Groupe de plaignants du procès des lépreux », a proposé qu'il la mette pour monter dans le train. Celui-ci a alors accepté et l'a portée. À cette époque, ces procès faisaient la une de tous les journaux et télévisions, et des informations sur le sujet étaient diffusées continuellement. À l'intérieur du train, des gens ont dit à monsieur Shibata de tenir bon. À chaque mot d'encouragement, monsieur Shibata répondait par des remerciements. En fait, monsieur Shibata était un plaignant

16. Voir également : Shibata, 1997. 


\section{CIPANGO}

30

Cahiers d'études japonaises $\mathrm{n}^{\circ} 24$

qui, après son passage dans les sanatoriums, avait réussi à s'intégrer au sein de la société au prix d'efforts considérables. Mais il m'a dit qu'à chaque question sur les symptômes qui persistaient suite à la lèpre, il mentait en disant qu'il s'agissait de stigmates de la bombe de Hiroshima (du fait de la radioactivité) ${ }^{17}$. C'est pour cela que ce moment où il a mis son écharpe qui portait l'inscription « Groupe de plaignants du procès des lépreux », ainsi que le moment où il est entré sans hésiter et a marché tête haute, sont des souvenirs inoubliables. J'y voyais une personne qui combattait et essayait de faire admettre à l'État, en mettant de côté sa dignité, les erreurs qui ont conduit à établir un régime au sein duquel de nombreuses vies ont subi de rudes épreuves. C'est un grand «trésor » pour l'auteur que d'avoir pu côtoyer de près ces plaignants, jour après jour.

\section{Bibliographie}

Fujıno Yutaka 藤野豊, 2001, «Inochi » no kindai-shi - «Minzoku jōka » no na no moto ni hakugai sareta hansenbyō kanja 「いのち」の近代史一「民族 浄化」の名のもとに迫害されたハンセン病患者 (L'histoire moderne de la « vie »- Les patients atteints de la maladie de Hansen qui ont été persécutés au nom de la « purification ethnique 》), Kamogawa shuppan かも がわ出版, Kyōto, p. 80-83.

HANSENBYŌ IKEN KOKUBAI SAIBAN ZENSHI HENSHŪ IINKAI ハンセン病違 憲国賠裁判全史編集委員会 (Comité de rédaction de l'histoire des procès en indemnisation des victimes des mesures d'isolement), 2006, Hansenbyō iken kokubai saiban zenshi dai 8 kan - Higai jittai-hen - Higashinihon soshō ハンセン病違憲国賠裁判全史第8巻一被害実態編一東日本訴訟 (Histoire des indemnisations des victimes des mesures d' isolement, Tome 8 La réalité des dommages - Procès de l'est du Japon), Kōseisha 皓星社, Tōkyō, p. 378-379.

17. Notons que les victimes de la bombe atomique ont aussi été l'objet de graves discriminations au Japon. 
HANSENBYŌ IKEN KOKUBAI SOSHŌ BENGODAN ハンセン病違憲国賠訴 訟弁護団 (Avocats des procès en indemnisation des victimes des mesures d'isolement), 2003, Hirakareta tobira - Hansenbyō saiban o tatakatta hitotachi 開かれた扉一ハンセン病裁判を闘った人たち (Portes ouvertes - Les personnes qui ont combattu dans le cadre des procès en indemnisation des victimes des mesures d'isolement), Kōdansha 講談社, Tōkyō, p. 226-227.

IsHIZAKI Manabu 石埼学, 2017, Hō chitsujo to shakai-teki shūen - Hansenbyōsha kakuri seisaku o rei to shite 法秩序と社会的周縁一ハンセン病者隔 離政策を例として (Ordre juridique et périphérie sociale - L'exemple de la politique de ségrégation des lépreux), Ryūkoku hōgaku 龍谷法学, no 49-4.

Matsubara Yōko, 2002, « Botai hogo hō no rekishi teki haikei »母体保護法 の歴史的背景 (L'arrière-plan historique de la loi sur la protection du corps maternel), in SAITō Yukiko 齋藤有紀子 (dir.), Botai hogohō to watashitachi : chüzetsu, tatai gensū, funin shujutsu o meguru seido to shakai 母体保護法 とわたしたち：中絶・多胎減数・不妊手術をめぐる制度と社会 (La loi sur la protection du corps maternel et nous : stérilisation, réduction embryonnaire, avortements dans les institutions et la société), Akashi shoten 明石書店, Tōkyō.

NICHIBENREN HŌMU KENKYŪ ZAIDAN 日弁連法務研究財団 (Fondation de recherches juridiques de la Fédération japonaise des associations du barreau), 2007, Hansenbyō mondai ni kansuru kenshō kaigi - Saishū hōkoku-sho ハン セン病問題に関する検証会議一最終報告書 (Réunion d'enquête sur le problème de la lèpre - Dernier rapport), 2 tomes, Akashi shoten, Tōkyō.

TAKAnami Atsushi 高波淳, 2003, Ikinuita! - Hansenbyō moto kanja no shōzō to kiseki 生き抜いた！一ハンセン病元患者の肖像と軌跡 (J'ai survécu！ Portraits et traces d'anciens lépreux), Sōfūkan 草風館, Tōkyō.

ShibAta Ryōhei柴田良平, 1997, Rokujūhassai no haru - kakuri kara no kaihō 六八歳の春一隔離からの解放 (Le printemps des 68 ans - La libération de l'isolement), Zenkoro ゼンコロ, Tōkyō. 
Résumé : En mai 2001, le tribunal de district de Tōkyō rendit une décision historique en reconnaissant la responsabilité de l'État dans les politiques d'isolement des lépreux menées sous la loi de prévention contre la lèpre. Cette décision permit de mettre en lumière l'étendue des dégâts malgré ses limites, à savoir le dommage collectif non individualisé. Ishizaki Manabu, constitutionnaliste, accompagna les victimes des politiques d'isolement tout au long du procès et en livre un témoignage précieux.

Mots-clés : lèpre, léproserie, Tama Zenshôen, politiques d'isolement, avortement, stérilisation

Abstract: In May of 2001, the Tōkyo district court handed in a historic decision by recognizing the State's responsibility in the enforcing of lepers' isolation policies within the scope of the Leper Prevention Law. This decision, though limited in part, shone a light on the extent of the damage the law had entailed the form of collective damage. Ishizaki Manabu, a Constitutionalist, assisted the victims of these isolation policies during the trial and delivers in this article a precious and poignant testimony. Keywords: Leper, leprosy, Tama Zenshôen, isolation policy, abortion, sterilization

$$
\text { キーワード：ハンセン病、らい病、ハンセン病療養所、多磨全生 }
$$
園、隔離政策、中絶、断種手術、優生手術 